\title{
HAMILTONIAN STRUCTURE OF A \\ FRIEDMANN-ROBERTSON-WALKER \\ UNIVERSE WITH TORSION
}

\author{
GIAMPIERO ESPOSITO
}

\begin{abstract}
Department of Applied Mathematics and Theoretical Physics, Silver Street, Cambridge CBз $9 E W, U . K$.

St. John's College, Cambridge CB2 1TP, U.K.
\end{abstract}

\begin{abstract}
Summary. - We study a $R^{2}$ model of gravity with torsion in a closed FriedmannRobertson-Walker universe. The model is cast in Hamiltonian form subtracting from the original Lagrangian the total time derivative of $f_{K} f_{R}$, where $f_{K}$ is proportional to the trace of the extrinsic curvature tensor, and $f_{R}$ is obtained differentiating the Lagrangian with respect to the highest derivative. Torsion is found to lead to a primary constraint linear in the momenta and a secondary constraint quadratic in the momenta, and the full field equations are finally worked out in detail. Problems to be studied for further research are the solution of these equations and the quantization of the model. One could then try to study a new class of quantum cosmological models with torsion.
\end{abstract}




\section{HAMILTONIAN STRUCTURE OF A FRW UNIVERSE WITH TORSION}

\section{1. - Introduction.}

A central issue in modern cosmology is the role played by theories of gravitation other than Einstein's general relativity in getting a better understanding of the early universe. Among the various alternative theories, the ones with torsion [1] are still receiving careful consideration. Most theories with torsion are such that spin can be thought of as the source of torsion. There are at least two very important motivations for studying this kind of gravitational theories with torsion (we are indebted to C. Stornaiolo for explaining these issues). In fact:

a) The holonomy theorems $[2,3]$ imply that torsion and curvature are related, respectively, to the groups of translations and of homogeneous transformations in the tangent vector spaces to a manifold. In general relativity the spin does not play any dynamical role, so that the infinitesimal holonomy group is given just by the homogeneous transformations. The introduction of torsion related to spin introduces a much stronger link between gravitation and particle physics, because it extends the holonomy group to the translations. A very enlightening discussion of gauge translations can be found in [4] (together with the literature given therein) and in [5]. In particular, the introduction of [5] makes clear from the very beginning the main geometric role played by the translations in the gauge group: they change a principal fibre bundle having no special relationship between the points on the fibres and the base manifold into the bundle of linear frames of the base manifold. A different view is expressed in sect. 5 of [6].

b) At the very high densities present in the early universe, the effects of spin are no longer negligible. In addition, theoretical investigations have shown that torsion may lead to the avoidance of the big-bang singularity in some cases (see for example [7]).

A good treatment of a theory where the sources of gravity are mass and spin is for example [8]. In that paper the Poincaré gauge theory of gravitation is studied, setting up a first-order Lagrangian formalism in a Riemann-Cartan space-time. However, in our paper we will not focus our attention on this kind of theories. In fact, we are more interested in getting a preliminary understanding of some basic features of the canonical structure of 


\section{HAMILTONIAN STRUCTURE OF A FRW UNIVERSE WITH TORSION}

$R+R^{2}$ theories of gravity with torsion. The reader should keep in mind that in theories with torsion derivatives higher than 2 are not present if frame and connection are regarded as independent variables. The $R^{2}$ terms arise already in the torsion-free case as quantum corrections to the effective action. In general such theories suffer from serious problems due to the presence of ghosts. However in [9-11] it has been found that some $R+R^{2}$ theories are ghost-free. This is indeed a delicate point. In fact in studying teleparallel theories (where the constraint of vanishing curvature is imposed), the authors in [12] found that the most general quadratic Lagrangian for such theories leads to dipole ghosts. These are field modes with free-field energies not bounded from below, and exist in view of $p^{4}$-poles. The authors in [12] also studied the general Poincaré gauge theory, and in sect. 5 of their paper they discussed in detail the difference between their work and the approach used in [9] in dealing with $p^{4}$-poles. Other reasons for studying $R+R^{2}$ theories with torsion are:

i) The calculation of the Hamiltonian for $R+R^{2}$ models with torsion is a very interesting field of research, because it provides an extremely fertile interplay between Dirac's theory of constrained Hamiltonian systems and the problems of gravitation and cosmology. Here we would like to mention the important paper [13], where Dirac's method is applied to the most general, parity-conserving Lagrangian, which is at most quadratic in the torsion and the curvature (the special $R^{2}$ case is treated in [14], whereas higher-order theories in the Riemannian case are studied for example in [15]).

ii) In the torsion-free case, $R+R^{2}$ theories have been shown to lead to an inflationary universe $[16,17]$. Moreover, other theories with torsion can lead to inflation (see for example $[18,19])$.

iii) In $R+R^{2}$ theories in the most general case, torsion may be expected to propagate, a remarkable property which, however, is not shared by the Einstein-Cartan-Sciama-Kibble theory based on spin.

In sect. $\mathbf{2}$ we study a $R^{2}$ model of gravity with torsion in a closed FriedmannRobertson-Walker (hereafter referred to as FRW) universe. The consideration of this model is suggested by a Lagrangian studied in [10]. We cast the model in Hamiltonian form using a canonical variable obtained differentiating the Lagrangian with respect to the highest derivative. Torsion makes itself manifest in that it induces one primary constraint 
$\phi_{1}$, linear in the momenta. Requiring the preservation in time of $\phi_{1}$, we end up with a secondary constraint $\phi_{2}$ quadratic in the momenta. Contact is also made with the canonical structure of the corresponding torsion-free model. In sect. 3 we summarize the results obtained, and we mention the problems to be studied for further research.

\section{2. - $R^{2}$ theories with torsion: a closed FRW model.}

We are here interested in a $R^{2}$ theory of gravity with torsion. The Lagrangian of our model is assumed to be given by $16 \pi G L_{g}=N \sqrt{h} \mu\left({ }^{(4)} R\right)^{2}$, where no assumption on the sign of $\mu$ is made. Indeed, a slightly more general Lagrangian would be

$$
16 \pi G L_{g}=N \sqrt{h}\left(\lambda\left({ }^{(4)} R\right)+\mu\left({ }^{(4)} R\right)^{2}\right)
$$

This model was at first studied by Rauch and Nieh [10], though not in a FRW universe and not using canonical techniques. Thus our Lagrangian is a special case of the Lagrangian studied in [10] when $\lambda=0$. In so doing our analytical derivations will be simplified, which in turn implies an advantage in trying to solve numerically the field equations, which will be shown to be rather involved. Moreover, setting $\lambda=0$ we realize a more direct comparison with the canonical structure of the torsion-free model studied in sect. $\mathbf{3}$ of [17]. Thus both our formalism and the physical content of our model are rather different from what has been discussed in [10]. The only basic ideas we strictly need to recall are the following. In a theory of gravity with torsion we have a four-dimensional space-time manifold with a metric tensor and a nonsymmetric linear connection $\Gamma_{\mu \nu}^{\lambda}$ which obeys the metricity condition [1]. The torsion tensor in a coordinate frame is defined by

$$
S_{\mu \nu}^{\lambda}=\frac{1}{2}\left(\Gamma_{\mu \nu}^{\lambda}-\Gamma_{\nu \mu}^{\lambda}\right)=-S_{\nu \mu}^{\lambda} .
$$

One also defines a contorsion tensor

$$
C_{\nu \mu}^{\lambda}=S_{\nu \mu}^{\lambda}-S_{\mu \nu}^{\lambda}+S_{\nu \mu}^{\lambda},
$$


so that

$$
\Gamma_{\mu \nu}^{\lambda}=\left\{\begin{array}{c}
\lambda \\
\mu \nu
\end{array}\right\}-C_{\nu \mu}^{\lambda}
$$

where $\left\{\begin{array}{c}\lambda \\ \mu \nu\end{array}\right\}$ are the Christoffel symbols. We shall use a convention according to which the curvature tensor is given by [20]

$$
R_{\mu \nu \beta}^{\alpha}=\Gamma_{\mu \nu, \beta}^{\alpha}-\Gamma_{\mu \beta, \nu}^{\alpha}+\Gamma_{\mu \nu}^{\lambda} \Gamma_{\lambda \beta}^{\alpha}-\Gamma_{\mu \beta}^{\lambda} \Gamma_{\lambda \nu}^{\alpha}
$$

We will focus our attention on a closed FRW universe. In view of the hypothesis of spatial homogeneity and isotropy, we are interested in a torsion tensor $S_{\mu \nu}{ }^{\lambda}$ whose only nonvanishing components are

$$
S_{10}^{1}=S_{20}^{2}=S_{30}^{3}=Q(t)
$$

In our model, the metric may be locally cast in the form

$$
d s^{2}=-N^{2}(t) d t^{2}+a^{2}(t)\left(d \chi^{2}+(\sin \chi)^{2} d \theta^{2}+(\sin \chi)^{2}(\sin \theta)^{2} d \phi^{2}\right) .
$$

Thus one finds (see appendix)

$$
{ }^{(4)} R=\frac{6}{a^{2}}+12\left(\frac{\dot{a}}{N a}\right)^{2}-\frac{72 \dot{a} Q}{a N^{2}}+\frac{96 Q^{2}}{N^{2}}+\frac{6}{N} \frac{d}{d t}\left(\frac{\dot{a}}{N a}-\frac{2 Q}{N}\right) \text {. }
$$

We now define

$$
I=\frac{\mu}{16 \pi G} \int_{M}\left({ }^{(4)} R\right)^{2} N \sqrt{h} d^{4} x .
$$

Therefore, putting $\beta=3 \pi / 2 G, \alpha=\lg (a), \widetilde{\mu}=\mu \beta$, we find

$$
\begin{gathered}
I=\int L d t \\
L=\frac{\widetilde{\mu}}{12} \exp [3 \alpha] N . \\
\cdot\left[6 \exp [-2 \alpha]+\frac{12 \dot{\alpha}^{2}}{N^{2}}-72 \frac{\dot{\alpha} Q}{N^{2}}+\frac{96 Q^{2}}{N^{2}}+\frac{6}{N} \frac{d}{d t}\left(\frac{\dot{\alpha}-2 Q}{N}\right)\right]^{2} .
\end{gathered}
$$


HAMILTONIAN STRUCTURE OF A FRW UNIVERSE WITH TORSION

Thus, putting

$$
\frac{Q}{N}=y, \quad \tau \int N d t,
$$

denoting by a prime the derivative with respect to $\tau$, and defining $\mu^{0}=3 \widetilde{\mu}$, we obtain the relations

$$
\begin{gathered}
I=\int \widetilde{L} d \tau \\
\widetilde{L}=\mu^{0} \exp [3 \alpha]\left[\exp [-2 \alpha]+2{\alpha^{\prime}}^{2}-12 y \alpha^{\prime}+16 y^{2}+\alpha^{\prime \prime}-2 y^{\prime}\right]^{2} .
\end{gathered}
$$

Now, in the torsion-free case (compare with [17]) one defines the variable $z \equiv \partial \widetilde{L} / \partial \alpha^{\prime \prime}$, and one considers the Lagrangian (we are indebted to D. Giulini for clarifying this point):

$$
L^{\prime} \equiv \widetilde{L}-\frac{d}{d \tau}\left(\alpha^{\prime} z\right)
$$

The geometrical meaning of $z$ is that it is proportional to the scalar curvature through $\widetilde{\mu} \exp [3 \alpha]$. In defining (2.14), we fix $\alpha$ and $z$ rather than $\alpha$ and $\alpha^{\prime}$ at the initial and final times. In our model with torsion, we can no longer use (2.14) as our final Lagrangian $L^{\prime}$. In fact, if one defines $p_{\alpha}=\partial L^{\prime} / \partial \alpha^{\prime}, p_{y}=\partial L^{\prime} / \partial y^{\prime}$ and $p_{z}=\partial L^{\prime} / \partial z^{\prime}$ using (2.14), the resulting relations do not involve $y^{\prime}$, whereas $p_{y}=-2 z$. Thus it is not possible to compute the Legendre transform: $H=p_{\alpha} \alpha^{\prime}+p_{z} z^{\prime}+p_{y} y^{\prime}-L^{\prime}$. However, we can point out that in (2.14) $\alpha^{\prime}$ is a function proportional to the trace of the extrinsic curvature tensor. In our model with torsion, $\alpha^{\prime}$ gets replaced by $\alpha^{\prime}-2 y$ (see (2.7) and (2.13)). This suggests to define

$$
L^{\prime} \equiv \widetilde{L}-\frac{d}{d \tau}\left[\left(\alpha^{\prime}-2 y\right) z\right]
$$

In deriving (2.15) in a more systematic way, we can point out that the total derivative appearing in (2.14) is

$$
\alpha^{\prime \prime} \frac{\partial \widetilde{L}}{\partial \alpha^{\prime \prime}}+\alpha^{\prime} \frac{d}{d \tau} \frac{\partial \widetilde{L}}{\partial \alpha^{\prime \prime}} .
$$


Thus, if torsion has to play a role, we expect having to add the term

$$
y^{\prime} \frac{\partial \widetilde{L}}{\partial y^{\prime}}+y \frac{d}{d \tau} \frac{\partial \widetilde{L}}{\partial y^{\prime}}=-2 \frac{d}{d \tau}(y z)
$$

so that we end up with definition (2.15). To sum up, in defining the correct $L^{\prime}$ we require that

a) it must be possible to compute the Legendre transform: $H=p_{\alpha} \alpha^{\prime}+p_{z} z^{\prime}+p_{y} y^{\prime}-L^{\prime}$;

b) $L^{\prime}$ must reduce to (2.14) in the torsion-free case, when $y=0$;

c) $L^{\prime}$ must not contain derivatives of $y$ higher than $y^{\prime}$, because these derivatives are absent in (2.13);

d) $L^{\prime}$ must be defined in a unique way, through a general method;

e) $L^{\prime}$ must be of the kind $L^{\prime} \equiv \widetilde{L}-(d / d \tau)\left(f_{K} f_{R}\right)$ where $f_{K}$ is the function $\alpha^{\prime}-2 y$, proportional to the trace $K$ of the full extrinsic curvature tensor, and $f_{R}$ is proportional through $\widetilde{\mu} \exp [3 \alpha]$ to the full scalar curvature ${ }^{(4)} R$.

It is also worth remarking that:

i) in view of (2.1)-(2.3) and (2.5), the only components of the connection (2.3) which depend both on $\alpha$ and on $Q$ are $\Gamma_{01}^{1}, \Gamma_{02}^{2}$ and $\Gamma_{03}^{3}$, and they are all equal to $\dot{\alpha}-2 Q=$ $N\left(\alpha^{\prime}-2 y\right)$;

ii) formula (2.15) can be written in the form

$$
L^{\prime} \equiv \widetilde{L}-\frac{d}{d \tau}\left(x^{\prime} \frac{\partial \widetilde{L}}{\partial x^{\prime \prime}}\right)
$$

which is formally of the same kind of (2.14), if we define $x=\alpha-2 \int y d \tau$;

iii) if we require that the constraint $p_{y} \approx 0$ should be avoided and that all constraints produced by torsion should be linear in the momenta, we are led to define

$$
L^{\prime} \equiv \widetilde{L}-\frac{d}{d \tau}\left[\alpha^{\prime}\left(z+\sum_{l=1}^{\infty} f_{l}(\alpha) y^{l}\right)\right]
$$

But in so doing there are infinitely many forms of $L^{\prime}$, one for each choice of the set of functions $\left\{f_{l}(\alpha)\right\}$, and our construction is completely arbitrary, violating requirement $\mathrm{d}$ ). 
Thus we will use (2.15), where, putting

$$
y=u^{\prime}
$$

one has

$$
z \equiv \frac{\partial \widetilde{L}}{\partial x^{\prime \prime}}=2 \mu^{0} \exp [3 \alpha]\left[\exp [-2 \alpha]+2{\alpha^{\prime}}^{2}-12 u^{\prime} \alpha^{\prime}+16{u^{\prime}}^{2}+\alpha^{\prime \prime}-2 u^{\prime \prime}\right]
$$

As one can easily check, putting $y=u^{\prime}$ one has the advantage of dealing with less involved calculations. Using the definitions $p_{\alpha}=\partial L^{\prime} / \partial \alpha^{\prime}, p_{z}=\partial L^{\prime} / \partial z^{\prime}, p_{u}=\partial L^{\prime} / \partial u^{\prime}$, we find that the effective Hamiltonian $\widetilde{H}$ is given by

$$
\widetilde{H}=H+\gamma \phi_{1} .
$$

In (2.18), one has

$$
\begin{aligned}
H & =p_{\alpha} \alpha^{\prime}+p_{z} z^{\prime}+p_{u} u^{\prime}-L^{\prime} \\
& =-4 z p_{z}^{2}+\frac{p_{z} p_{u}}{2}+\frac{z^{2} \exp [-3 \alpha]}{4 \mu^{0}}-z \exp [-2 \alpha]
\end{aligned}
$$

with the primary constraint

$$
\phi_{1}=p_{u}+2 p_{\alpha}-4 z p_{z}
$$

The constraint $\phi_{1} \approx 0$ must now be preserved in time using Dirac's method [21] for constrained Hamiltonian systems. Namely, computing the Poisson bracket of $\phi_{1}$ with $\widetilde{H}$, we find the secondary constraint:

$$
\phi_{2}=16 z p_{z}^{2}-2 p_{u} p_{z}+\frac{7 z^{2}}{2 \mu^{0}} \exp [-3 \alpha]-8 z \exp [-2 \alpha]
$$

In other words, torsion introduces a primary constraint $\phi_{1}$ linear in the momenta, which in turn leads to one more secondary constraint $\phi_{2}$ quadratic in the momenta. Finally, so as to preserve in time the constraint $\phi_{2} \approx 0$, we require that the following Poisson bracket must vanish:

$$
\left\{\phi_{2}, \widetilde{H}\right\}=\left\{\phi_{2}, H\right\}+\gamma\left\{\phi_{2}, \phi_{1}\right\}
$$


Setting the right-hand side of (2.22) equal to zero and solving for $\gamma$ we find

$$
\gamma=-\frac{\left\{\phi_{2}, H\right\}}{\left\{\phi_{2}, \phi_{1}\right\}}
$$

where

$$
\begin{gathered}
\left\{\phi_{2}, \phi_{1}\right\}=64 z p_{z}^{2}-8 p_{u} p_{z}-\frac{49 z^{2}}{\mu^{0}} \exp [-3 \alpha]+64 z \exp [-2 \alpha] \\
\left\{\phi_{2}, H\right\}=\left(96 \exp [-2 \alpha] z-\frac{72 \exp [-3 \alpha]}{\mu^{0}} z^{2}\right) p_{z} \\
+\left(\frac{9 \exp [-3 \alpha]}{2 \mu^{0}} z-6 \exp [-2 \alpha]\right) p_{u}
\end{gathered}
$$

Using the constraint $\phi_{1} \approx 0$, we can also cast (2.19) in the form

$$
H=-2 z p_{z}^{2}-p_{\alpha} p_{z}+\frac{z^{2} \exp [-3 \alpha]}{4 \mu^{0}}-z \exp [-2 \alpha] .
$$

Thus the equations of motion of our model are given by

$$
\begin{gathered}
\alpha^{\prime}=\frac{\partial \widetilde{H}}{\partial p_{\alpha}}=-p_{z}+2 \gamma, \\
z^{\prime}=\frac{\partial \widetilde{H}}{\partial p_{z}}=-4 z p_{z}-p_{\alpha}-4 \gamma z, \\
u^{\prime}=\frac{\partial \widetilde{H}}{\partial p_{u}}=\gamma, \\
p_{\alpha}^{\prime}=-\frac{\partial \widetilde{H}}{\partial \alpha}=\frac{3 z^{2}}{4 \mu^{0}} \exp [-3 \alpha]-2 z \exp [-2 \alpha], \\
p_{z}^{\prime}=-\frac{\partial \widetilde{H}}{\partial z}=2 p_{z}^{2}-\frac{z \exp [-3 \alpha]}{2 \mu^{0}}+\exp [-2 \alpha]+4 \gamma p_{z}, \\
p_{u}^{\prime}=-\frac{\partial \widetilde{H}}{\partial u}=0,
\end{gathered}
$$


plus the constraints: $\phi_{1} \approx 0, \phi_{2} \approx 0$ and the formula for $\gamma($ see $(2.19)-(2.21)$ and (2.23)$(2.25))$. In other words, we deal with a coupled system of six first-order ordinary differential equations subject to constraints. A remarkable result is that we have written the field equations in Hamiltonian form without having to solve any differential equation involving torsion. Moreover, (2.32) implies that $p_{u}=$ const. It is also very important remarking that, imposing the particular value $H=0$ (see (2.19)), we can solve for $z p_{z}^{2}$. Inserting this relation into the constraint $\phi_{2}=0$, we obtain the relation

$$
\exp [\alpha]-\frac{3}{8 \mu^{0}} z=0
$$

In other words, requiring the constraints $\phi_{1}=0, \phi_{2}=0$ and setting $H=0$ is equivalent to require that $\Phi_{1}, \Phi_{2}$ and $H$ must vanish, where $\Phi_{1}=\phi_{1}$ and $\Phi_{2}$ is given by the left-hand side of (2.33). The constraints $\phi_{1}$ and $\phi_{2}$ are second class (they cannot be brought into the first class by suitable linear combinations [21]). It is also worth emphasizing that in the torsion-free case no primary constraints such as $\phi_{1}$ are found to arise. Setting $y=0$ in (2.13) and using (2.14) one finds then

$$
\begin{gathered}
\alpha^{\prime}=-p_{z}, \\
z^{\prime}=-4 z p_{z}-p_{\alpha}, \\
p_{\alpha}^{\prime}=\frac{3 z^{2}}{4 \mu^{0}} \exp [-3 \alpha]-2 z \exp [-2 \alpha], \\
p_{z}^{\prime}=2 p_{z}^{2}-\frac{z}{2 \mu^{0}} \exp [-3 \alpha]+\exp [-2 \alpha],
\end{gathered}
$$

plus the particular value for the Hamiltonian here chosen

$$
2 z p_{z}^{2}+p_{\alpha} p_{z}+z \exp [-2 \alpha]-\frac{z^{2}}{4 \mu^{0}} \exp [-3 \alpha]=0
$$

So as to avoid confusion, it is important to remark that, using our notation, one has

$$
\alpha^{\prime}=\frac{\dot{a}}{N a}, \quad \alpha^{\prime \prime}=\frac{1}{N} \frac{d}{d t}\left(\frac{\dot{a}}{N a}\right)
$$


whereas in other papers such as [17] one defines

$$
\begin{gathered}
\alpha^{\prime}=\frac{d \alpha}{d \eta}=\frac{d \alpha}{d t} \frac{d t}{d \eta}=a \dot{\alpha}=\dot{a} \\
\alpha^{\prime \prime}=\frac{d \alpha^{\prime}}{d \eta}=a \frac{d \alpha^{\prime}}{d t}=a \ddot{a} .
\end{gathered}
$$

System (2.27)-(2.32) with the constraints (2.20)-(2.21) is very difficult, and at the classical level we have not a criterion which picks out a preferred choice of initial conditions. Indeed, exact solutions for theories with torsion are known for a large class of theories. An important recent paper is [22], where the authors have studied the Hamiltonian structure of Poincaré gauge theory. In the case of spherical symmetry and for the charged TaubNUT metric, the authors of [22] have obtained the most general torsion configuration for a large class of quadratic Lagrangians (see also references in [22] for related work). However, our original aim was just the study of the canonical structure of the model described by (2.5)-(2.6) and (2.8) at the classical level. Thus we prefer to stop here our analysis, and to outline in detail the unsolved issues in the next section.

\section{3. - Conclusions.}

In this paper we have studied a Hamiltonian approach to a closed FRW universe with torsion. The consideration of our $R^{2}$ Lagrangian (see (2.8)) has been suggested by a model at first studied by Rauch and Nieh [10]. Our main results are the following:

1) the technique used by Horowitz [17] in the torsion-free case can be generalized using (2.15)-(2.17);

2) torsion modifies the canonical structure of the theory in that it induces a primary constraint $\phi_{1}$ linear in the momenta and a secondary constraint $\phi_{2}$ quadratic in the momenta (see (2.20)-(2.21));

$3)$ the field equations are much more complicated, and are given by (2.27)-(2.32). 


\section{HAMILTONIAN STRUCTURE OF A FRW UNIVERSE WITH TORSION}

Thus the most remarkable feature of our model seems to be the appearance of the additional constraints $\phi_{1}$ and $\phi_{2}$, or equivalently $\Phi_{1}=\phi_{1}$ and $\Phi_{2}$ (see (2.33)). In general relativity, the secondary constraints reflect the invariance of the theory under fourdimensional diffeomorphisms. Thus in our model we can say that torsion introduces an additional invariance under four-diffeomorphisms, leading to $\phi_{2}$, which contributes to the generation of the dynamics through $\gamma$ (see (2.23)-(2.25) and (2.27)-(2.32)). In the as yet undeveloped minisuperspace model, we expect the physical state $\psi$ will still be a function of the three-metric $h_{i j}$ and of the extrinsic curvature $K_{i j}$ as in the torsion-free case [17]. In fact, the nonvanishing part of torsion is in our case just the one contained in $K_{(i j)}$ (see appendix of [20]), and from (2.27) and (2.29) one has $\alpha^{\prime}-2 u^{\prime}=\alpha^{\prime}-2 y=-p_{z}$, which is formally identical to (2.34), because in our model with torsion the trace $K$ is proportional to $\alpha^{\prime}-2 y$ (see sect. 2). Indeed, Hamiltonian methods had also been used for the canonical quantization of more complicated $R^{2}$ theories of gravity in the torsion-free case [23,24], and in studying spherically symmetric gravitational fields with electromagnetism and a massless scalar field as sources [25]. For theories with torsion, a rather important paper is [26], and in fact the ansatz (2.5) of our paper is closely related to the ansatz studied in sect. 2 and sect. 6 of [26]. A minor difference between our work and [26] lies in the different parametrization of the FRW metric, and in the fact the authors of [26] set the lapse function equal to one, whereas we keep it arbitrary. But the main difference is the following: the authors of [26] do not perform a canonical analysis, and thus they do not deal with six first-order equations subject to constraints. A comparison with their analytical derivation has not yet been possible because it is very difficult to solve our equations of motion. Therefore, the main problems to be studied for further research are the choice of the initial conditions for the numerical integration of the field equations, the quantization of the model using path integrals and the attempt of applying the Hartle-Hawking proposal [27] or other proposals. In fact we cannot solve the field equations without knowing the initial conditions. Quantum cosmology can provide a way for doing so, but then one has to work out the path-integral quantization, discussing in detail the measure in the path integral, the metrics we are summing over, the gauge fixing and the ghost action (namely the action involving Faddeev-Popov ghosts). Moreover, it is necessary to understand whether 


\section{HAMILTONIAN STRUCTURE OF A FRW UNIVERSE WITH TORSION}

the underlying quantum theory is affected by negative-norm states, whose occurrence can be a serious drawback. The quantization procedure is here complicated by the additional constraints $\Phi_{1}$ and $\Phi_{2}$.

Indeed, torsion is much less ad hoc than the fundamental scalar fields considered so far in cosmological models (for the role played by scalar fields, see for example [28,29]), though its existence is still to be proved. Moreover, in the torsion-free case $R+R^{2}$ theories may play an important role not only for the inflationary era, or in giving a self-consistent probabilistic interpretation of the wave function of the universe [30], but also in completely different processes such as black-hole evaporation [31]. This is why we think it is important to try to solve the above-mentioned problems. To be honest, we also must say that it is not yet clear to which extent we can use a closed FRW metric so as to study the early universe. Therefore the model we have studied is just a mathematical idealization. Nevertheless, the derivations obtained show that it is worth studying theories of gravity with torsion in further detail from a Hamiltonian point of view. We hope that our paper can stimulate further interaction between canonical gravity, theories with torsion and quantum cosmology. We think it would be also very useful to study the singularity problem for $R^{2}$ theories with torsion both in the isotropic and in the anisotropic case.

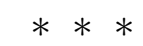

We are grateful to P. D'Eath, D. Giulini and J. Louko for useful conversations and to St. John's College for financial support.

APPENDIX

We are interested in the formula for the scalar curvature ${ }^{(4)} R$ for a theory of gravity with torsion. This kind of calculation is performed in detail in [32]. However, this reference 


\section{HAMILTONIAN STRUCTURE OF A FRW UNIVERSE WITH TORSION}

is not easily available. Thus we prefer to rely on the appendix of [20]. In that appendix, Pilati works out the Lagrangian density ignoring a total divergence. Now, in general form, and bearing in mind eqs. (A.16)-(A.18) of [20], we can write

$$
\begin{aligned}
{ }^{(4)} R & =K_{(a b)} K^{(a b)}-K_{[a b]} K^{[a b]}+K^{2}-2 \tau_{(a b)} K^{(a b)}+2 \tau_{[a b]} K^{[a b]}+2 K \tau-2 \frac{\dot{K}}{N} \\
& +{ }^{(3)} R+A+B+C
\end{aligned}
$$

where

$$
\begin{gathered}
A=h^{i j}\left[\left(\left(C_{m i}^{m}\right)_{, j}-\left(C_{j i}{ }^{m}\right)_{, m}+C_{j i}{ }^{l} C_{m l}{ }^{m}-C_{m i}{ }^{l} C_{j l}{ }^{m}\right)\right. \\
\left.+{ }^{(3)}\left\{\begin{array}{c}
l \\
i m
\end{array}\right\} C_{j l}{ }^{m}+{ }^{(3)}\left\{\begin{array}{c}
m \\
l j
\end{array}\right\} C_{m i}{ }^{l}-{ }^{(3)}\left\{\begin{array}{c}
l \\
i j
\end{array}\right\} C_{m l}{ }^{m}-{ }^{(3)}\left\{\begin{array}{c}
m \\
l m
\end{array}\right\} C_{j i}{ }^{l}\right], \\
B=-\frac{2}{N}\left(h^{i j} N_{\mid i j}-N^{i} K_{, i}\right), \\
C=-\rho^{a} q_{a}+2 \rho^{a}{ }_{\mid a}-2 \rho^{a} \rho_{a} .
\end{gathered}
$$

In (A.4), $\rho^{a}$ and $q_{a}$ are the ones defined in (A.30)-(A.31) of [20]. Using (2.5)-(2.6) and (A.1)-(A.4) of our appendix, one finds (2.7). Other useful references for the Hamiltonian formulation of ECSK theory are [33-35]. Finally, we wish to remark that, putting

$$
S=\frac{2 h^{k i} S_{k 0 i}}{N},
$$

(A.1) leads to a surface term involving torsion in the action integral

$$
\frac{1}{8 \pi G} \int_{\partial M} S \sqrt{h} d^{3} x
$$

in addition to the usual one of Einstein's theory [36-38]. 
HAMILTONIAN STRUCTURE OF A FRW UNIVERSE WITH TORSION

REFERENCES

[1] F. W. HEHL, P. von der HEYDE, G. D. KERLICK and J. M. NESTER: Rev. Mod. Phys., 48, 393 (1976).

[2] A. TRAUTMAN: Symp. Math., 12, 139 (1973).

[3] C. STORNAIOLO: Ph. D. Thesis (University of Naples, 1987).

[4] E. A. LORD and P. GOSWAMI: J. Math. Phys. (N.Y.), 29, 258 (1988).

[5] P. K. SMRZ: J. Math. Phys. (N.Y.), 28, 2824 (1987).

[6] D. IVANENKO and G. SARDANASHVILY: Pramana, 29, 21 (1987).

[7] M. DEMIANSKI, R. DE RITIS, G. PLATANIA, P. SCUDELLARO and C. STORNAIOLO: Phys. Lett. A, 116, 13 (1986).

[8] J. D. Mc CREA: in Differential Geometric Methods in Mathematical Physics, in Proceedings of the XIV International Conference, edited by P. L. GARCIA and A. PÉREZ-RENDÓN (Springer-Verlag, Berlin, 1987), p. 222.

[9] E. SEZGIN and P. van NIEUWENHUIZEN: Phys. Rev. D, 21, 3269 (1980).

[10] R. RAUCH and H. T. NIEH: Phys. Rev. D, 24, 2029 (1981).

[11] M. GASPERINI: Phys. Lett. B, 205, 517 (1988).

[12] R. KUHFUSS and J. NITSCH: Gen. Rel. Grav., 18, 1207 (1986).

[13] I. A. NIKOLIC: Phys. Rev. D, 30, 2508 (1984).

[14] V. SZCZYRBA: Phys. Rev. D, 36, 351 (1987).

[15] V. SZCZYRBA: J. Math. Phys. (N.Y.), 28, 146 (1987).

[16] S. W. HAWKING and J. C. LUTTRELL: Nucl. Phys. B, 247, 250 (1984).

[17] G. T. HOROWITZ: Phys. Rev. D, 31, 1169 (1985).

[18] R. DE RITIS, P. SCUDELlARO and C. STORNAIOLO: Phys. Lett. A, 126, 389 (1988).

[19] A. J. FEnNElly, J. C. BRADAS and L. L. SMALLEY: Phys. Lett. A, 129, 195 (1988).

[20] M. PILATI: Nucl. Phys. B, 132, 138 (1978). 
HAMILTONIAN STRUCTURE OF A FRW UNIVERSE WITH TORSION

[21] P. A. M. DIRAC: Lectures on Quantum Mechanics (Belfer Graduate School of Science, Yeshiva University, New York, N.Y., 1964).

[22] P. BAEKLER and E. W. MIELKE: Fortschr. Phys., 36, 549 (1988).

[23] D. BOULWARE in: Quantum Theory of Gravity, edited by S. M. CHRISTENSEN (Adam Hilger, Bristol, 1984), p. 267.

[24] I. L. BUCHBINDER and S. L. LYAHOVICH: Class. Quantum Grav., 4, 1487 (1987).

[25] B. K. BERGER, D. M. CHITRE, V. E. MONCRIEF and Y. NUTKIN: Phys. Rev. $D, \mathbf{5}, 2467$ (1972).

[26] H. GOENNER and F. MULLER-HOISSEN: Class. Quantum Grav., 1, 651 (1984).

[27] J. B. HARTLE and S. W. HAWKING: Phys. Rev. D, 28, 2960 (1983).

[28] S. W. HAWKING: Nucl. Phys. B, 239, 257 (1984).

[29] G. ESPOSITO and G. PLATANIA: Class. Quantum Grav., 5, 937 (1988).

[30] M. POLLOCK: Nucl. Phys. B, 306, 931 (1988).

[31] R. C. MYERS and J. Z. SIMON: Phys. Rev. D, 38, 2434 (1988).

[32] O. ALVAREZ: Senior Thesis (Princeton University, Princeton, N.J., 1974).

[33] L. CASTEllani, P. van NIEUWENHUIZEN and M. PIlAti: Phys. Rev. D, 26, 352 (1982).

[34] R. DI STEFANO and R. T. RAUCH: Phys. Rev. D, 26, 1242 (1982).

[35] J. ISENBERG and J. NESTER: in General Relativity and Gravitation, edited by A. HELD (Plenum Press, New York, N.Y., 1980), p. 23.

[36] J. W. YORK: Phys. Rev. Lett., 28, 1082 (1972).

[37] G. W. GIBBONS and S. W. HAWKING: Phys. Rev. D, 15, 2752 (1977).

[38] J. W. YORK: Found. Phys., 16, 249 (1986). 